\title{
Ileostomy or ileorectal anastomosis for ulcerative colitis?
}

\author{
British Medical fournal, 1978, 1, 1459-1463
}

Mr Peter Jones was asked by the $B M \mathcal{F}$ to present the case for treating patients with ulcerative colitis warranting colectomy by ileorectal anastomosis rather than by ileostomy. His article, published below, was then sent to two other surgeons and all three met to discuss the topic with one of the $B M \mathcal{F}$ medical editors, Dr Tony Smith, acting as chairman.

\section{Working paper}

When the patient with colitis requires surgical treatment panproctocolectomy is the operation which is generally performed, and most patients make a satisfactory adjustment to ileostomy, greatly helped by recent advances in stoma care. Nevertheless, an operation which leaves a permanent ileostomy must be regarded as a less than ideal solution, the more so when so many of the patients are young and on the threshold of marriage, higher education, or the start of a career. The alternative operation, ileorectal anastomosis after excision of the whole colon, has not yet received any general approval, probably because experts have expressed such widely differing views about its results. A close study of a personal series assembled over 20 years, and a review of the published reports, has led me to the opinion that, while there are a number of patients for whom preservation of the rectum is impossible or ill-advised, there is another group who can obtain a very good long-term result from ileorectal anastomosis.

\footnotetext{
Royal Aberdeen Children's Hospital, Aberdeen

PETER F JONES, MCH, FRCS, reader in paediatric surgery and consultant surgeon
}

Dudley Road Hospital, Birmingham

P GILROY BEVAN, CHM, FRCS, consultant surgeon

St Mark's Hospital for Diseases of the Rectum, London PETER R HAWLEY, MS, FRCS, consultant surgeon

\begin{abstract}
Personal series
This now consists of 94 consecutive patients who have undergone total colectomy for inflammatory disease of the colon. A provisional choice between excision or retention of the rectum was made preoperatively: whenever the anal canal appeared normal, and the rectum appeared normal or only mildly inflamed and capable of distension, then the patient was considered to be a candidate for ileorectal anastomosis. In a borderline case conservation of the rectum tended to be chosen if the patient was aged under 25 . The level of division of the rectum was decided at operation, after direct inspection of the mucosa, but commonly $12-15 \mathrm{~cm}$ of rectum was retained.

Forty patients $(42 \%)$ had an ileorectal anastomosis performed, 38 at total colectomy and two as a second-stage procedure after emergency total colectomy and ileostomy. At the time of operation all these patients were believed to have ulcerative colitis but a review of histology has shown that four of the earlier patients in fact had Crohn's colitis. A direct end-to-end anastomosis of ileum to rectum was always used and in no case was a covering ileostomy created.
\end{abstract}

\section{Results}

Thirty-eight patients made a good initial recovery from operation and two died; one patient had an emergency colectomy and anastomosis which leaked, and the patient died from uncontrolled sepsis two weeks later. (It was a serious error of judgment to attempt an anastomosis at emergency colectomy and this has never been done again. This was the only patient to develop an anastomotic leak). The other patient, who was gravely ill and taking corticosteroids, died two weeks after operation from uncontrolled sepsis.

Four patients have required subsequent ileostomy. One developed early postoperative intestinal obstruction and the anastomosis had to be taken down in the course of the operation; three developed a severe recurrence of proctitis requiring excision of the rectum; two of these patients had ulcerative colitis and one had Crohn's disease.

Of the 34 remaining patients, five have died since their operation. One of these had an excellent result for six years and then developed chronic active hepatitis, dying two years later. 
The other four died from causes not connected with the ileorectal anastomosis. All of the 29 surviving patients are working and living normal lives. Thirteen have up to three stools per day, and a further 12 have four to six stools per day; only one has over seven stools per day and no one is incontinent. Three have had continued proctitis and required medical treatment for this. Twenty-four of the 25 patients treated more than one year ago have had a recent sigmoidoscopy with multiple biopsy of the rectal stump: in six the mucosa looked normal, in 13 there was mild inflammation, and the remainder showed obvious signs of inflammation. The biopsy appearances were graded by an experienced pathologist who considered that those for 14 patients were normal or mildly inflamed. All biopsy specimens were closely scrutinised for precancer but none was found.

\section{Comment}

A review of published reports ${ }^{1}$ shows that the criteria used for adopting ileorectal anastomosis vary very widely, as do the results. Nevertheless, most surgeons who have reported their results have used ileorectal anastomosis in $30-40^{\circ}{ }_{0}$ of their patients who undergo elective total colectomy with an operative mortality of around $3^{\circ}{ }_{0}$-which is similar to that for elective proctocolectomy. There is a wide range in the postoperative leak rate but everyone experiences a high incidence of adhesive obstruction, as happens after proctocolectomy. The late results, as judged by the good health of the patient and the absence of rectal complaints, suggest that a good result is obtained by

\section{Discussion}

Chairman: While major textbooks still state (quite dogmatically) that "most experts agree that preservation of the rectum is not possible in ulcerative colitis," opinion does seem to have changed in the past 10 years in Britain. Could you, $\mathrm{Mr}$ Jones, explain why the change has come about?

Mr JONES: Probably a few people have always thought that ileorectal anastomosis was worth doing. For many years $\mathrm{Mr}$ Stanley Aylett at the Gordon Hospital seems to have been able to use the technique in almost all his cases. We started to do ileorectals in Aberdeen 20 years ago. This was inspired by my having seen Mr Lloyd-Davies at the Middlesex Hospital do a total colectomy and an ileorectal anastomosis on several patients with ulcerative colitis with what seemed very good results. I had been brought up, of course, on the thought that you couldn't save the rectum; so I was very favourably impressed and decided, when I became a consultant, to try the operation and see how it worked out.

Even so, some surgeons were quite vocal that it was not a good operation, not so much because of the risk of carcinoma in the rectal stump as because of fear that the rectum would become more inflamed with intolerable symptoms of diarrhoea and tenesmus. I remember a discussion at St Mark's in about 1955 when a senior surgeon said that it was an impossible operation: he'd tried it twice and on both occasions the result had been absolutely disastrous. Most of the opposition came from surgeons who had seen some very poor results.

Mr Bevan: I was brought up in the Bryan Brooke school, and in those days we had a lot of very desperate cases to deal with. One change since then is that we're seeing patients at an earlier stage before the disease is so advanced. But in my own series I've managed to do ileorectal anastomoses in only about $10^{\circ}{ }_{0}^{\circ}$ of the patients, and in those $10^{\circ}$ I've had to remove the rectum and convert to an ileostomy in about half.

Chairman: What about you, Mr Hawley. How has policy changed at St Mark's over the years?

Mr Hawley: From 25 years ago up until about 1970 the proportion of patients given an ileorectal anastomosis was about $10-15 \%$. From 1970 onwards we have been doing more; about $50-60^{\circ}{ }_{0}$ of all those undergoing ilcorectal anastomosis. The need for late rectal excision varies widely but in the majority of reports lies between $10^{\circ}{ }^{\circ}$ and $20^{\circ}{ }_{1}$.

The most important late complication is the development of a carcinoma in the rectal stump and there is no question thap the risk of this is real though infrequent. So far, collecting alt available reports, it has affected 1 in 30 of the patients under $\overline{0}$ going ileorectal anastomosis. There is no question that this. risk means that every patient must have an annual sigmoidoscop with rectal biopsies.

In conclusion, one cannot but be impressed by the good healt and normal lives of the patients with good results. Twenty of ou ㅜㅡㄹ patients were under 23 years of age at operation and the advan tage of the operation so far to them is very clear. Happily the highest proportion of successes with ileorectal anastomosi £ seems to be among younger patients. The disadvantages must be neither underrated nor overemphasised. Every patient must accept an annual sigmoidoscopy and the failure rate probabl $\vec{\omega}$ runs around $20^{\circ}{ }_{0}$, with $10-15^{\circ}{ }_{0}$, requiring later rectal excision? The risk of the development of a carcinoma in the rectal stump is the major worry because, while late relapse cannot be over: looked by the patient who defaults from follow-up, the developor ment of a carcinoma may easily go unnoticed. This is why regular follow-up is essential. Provided this is accepted, our considered opinion is that ileorectal anastomosis should continue to be offered to carefully selected patients.

${ }^{1}$ Jones, P F, Munro, A, and Ewen, S W B, British fournal of Surgery, $1977^{c}$ 64, 615 .

one-third of all cases that come to surgery have either a one or a two-stage ileorectal anastomosis. Some patients who have्ष quiescent colitis and an elective operation can have a one-stage procedure; but for those who come to surgery urgently I would not do an ileorectal anastomosis. Even so, I would not excise the rectum in an urgent operation, for some of these patients are suitable for a second-stage ileorectal later. For example, I car remember a girl who presented with two perforations, one in her caecum and one in her rectum, who had an emergency totak colectomy, the rectal perforation being oversewn from within Six months later she had an ileorectal anastomosis; and she has been one of our most successful patients, with only one bowe? action in 24 hours.

\section{Assessment at operation}

MR JONES: There is a curious paradox here. In fact you can' really relate prognosis to the appearance of the rectum at the time of operation. A patient with a good, quiet-looking rectuno who ought to make an excellent subject for ileorectal anastomosis may do badly. Others with rectums which really don't loop very promising at all but in whom, for one reason or another, one uses an ileorectal anastomosis, may do well.

Mr Bevan: My experience has been rather different. $\mathrm{Mr}_{r}^{\stackrel{\rho}{+}}$ Hawley has just advocated leaving the rectum when doing ano emergency colectomy for proctocolitis. I had three cases some years ago in which we did this, where the main symptom wa rectal haemorrhage, and in all three we had to go back an $\$$ remove the rectum in a short time because of continued bleeding요 Are these a special group ? Mr Hawley, do you think that where the main troubie is haemorrhage the rectum should come ouf in the emergency stage?

MR HAWLEY: Yes, I agree-but such cases are uncommon? I don't think one needs always to remove the rectum because् of this fear; but in patients who come to surgery urgently because of bleeding proctocolectomy is indicated. In the last seven or eight years we have had to go back urgently and remove 
the rectum in two patients because of bleeding-but that's two out of quite a large number of patients.

Chairman: May there be other reasons for the increasing use of ileorectal anastomosis beside a change in surgical attitudes ? Possibly the natural history of the disease has changed; possibly medical treatment has become more effective; possibly, too, in centres in which it is known that ileorectal anastomosis will be done if it is at all possible physicians are willing to refer their patients rather sooner.

Mr JONES: Certainly I am conscious of the fact that on several occasions I have felt able to advise ileorectal anastomosis for a child who couldn't go to school or an older person who

"Patients die from ulcerative colitis because they are operated on as emergencies instead of as elective cases"-Mr Peter Hawley.

couldn't work-but who still in a sense was not at a stage when operation was essential. These patients were in a state of invalidism; and the alternatives were to persevere with medical treatment in the hope that there would still be an improvement or to elect for surgery. It was very much easier to be able to recommend total colectomy and ileorectal anastomosis than to have to introduce them to the idea of a permanent ileostomy. In the long run those individuals have, I believe, benefited greatly from that decision.

MR Bevan: Indeed, the problem is no longer black and white in that some of us do only ileostomies and some of us do only ileorectal anastomosis. We've now reached the stage where most surgeons consider the best treatment for the individual patient. The difference between us is that $\mathrm{Mr}$ Jones clearly does a very much higher proportion of colectomies and ileorectal anastomoses than I do, with $\mathrm{Mr}$ Hawley perhaps holding an intermediate position.

MR HAWleY: At St Mark's most of our operations are elective or urgent rather than emergencies. We need to remember, however, that as a profession we have been obsessed by cancer in ulcerative colitis. This is rare, though it does occur, and it does not kill many patients. In Britain as a whole patients die from ulcerative colitis because they are operated on as emergencies instead of as elective cases. Statistics from the North-east Thames Regional Board (compiled by Dr Jean Ritchie at St Mark's) have shown that the mortality rate from operating on urgent cases is still $40 \%$. This high mortality rate is often attributable to surgeons being sent the patient too late. Next, I think that mortality is also increased by surgeons trying to do a protocolectomy in these circumstances with the increased chance of perineal and pelvic sepsis.

MR BEVAN: I think we have a better overall idea of the disease today. Physicians-particularly as there are so many more specialist gastroenterologists-are now picking up the disease earlier and making a definite diagnosis of ulcerative colitis. We no longer see patients who have been in a fever hospital for days or weeks being treated for salmonella infection.

\section{Follow-up clinics}

Chairman: Many people would be interested to know, $\mathrm{Mr}$ Jones, what sort of problems you have in running a follow-up clinic with a fairly large number of patients who have been treated by ileorectal anastomosis.

MR JONEs: The surgeon has a responsibility to make it clear to his patients that the annual follow-up is important. We've only one patient (who was operated on a long time ago) who just steadfastly refuses to have a follow-up sigmoidoscopy done: fortunately she seems to be going along on a perfectly even keel and is quite well. Undoubtedly, of course, if you perform a proctocolectomy with permanent ileostomy for ulcerative colitis you can more or less dismiss the patient after a year or two, if they have no problems. There is the difficulty, particularly with young people, that they are quite likely to move with their work, or on marriage, and this can make follow-up difficult. Even so, there are two sides to this. If they should elect to go abroad the difficulties and expense of running an ileostomy and the apparatus required are not to be entirely overlooked. On the other hand, if they are going to live somewhere else, they have to be told they are not completely free of disease: a patient with an ileorectal anastomosis should certainly attend for annual review, with sigmoidoscopy.

Mr HAwley: I-and my colleagues at St Mark's-always tell patients before they're operated on that they do need to be followed up. They still have active rectal disease, so I don't use the term cured or not cured. In a patient who has had an ileorectal anastomosis for ulcerative colitis the rectum is always diseased, and he may need treatment for this. We believe that patients with ileorectal anastomoses need to be seen at least once a year, usually once every six months. We like to monitor the rectum with mucosal biopsies: we believe that by picking out patients who develop severe dysplasia we can determine those who are going to develop cancer. There have been three patients in whom carcinoma has developed in the last few years and all were picked up at a very early stage. Two had cancers that were undetectable clinically. Two of the three are still alive over five years after excision of the rectum, so they are probably cured, and the other patient is alive without recurrence $2 \frac{1}{2}$ years after operation. Three further patients have had their rectums excised for severe dysplasia but without invasive carcinoma.

But essentially, whether one agrees with the philosophy of an ileorectal procedure or not depends on one's overall view of the patient's life. For a young person who is averse to having an ileostomy, it may help to have an ileorectal anastomosis even for a few years while he finishes his schooling, goes to university, perhaps marries and starts a family. I regard the operation as being a great success even if the ileorectal has to be taken down and the rectum excised, say, after ten years.

\section{Value of cure}

Mr Bevan: My own opinion doesn't quite agree with the two of you. In ulcerative colitis the big difference between proctocolectomy and ileostomy and colectomy and preservation of the rectum is that the first of these cures the patient and the second does not. Mr Hawley has said, rightly, that the development of carcinoma in the rectal stump is rare, and it is, and $\mathrm{Mr}$ Jones says that he aims at an annual sigmoidoscopy, though even that is really not adequate to detect an early carcinoma in the stump. But that's a small part of the problem. A patient with an ileorectal anastomosis and the rectum preserved is still living with the possibility of the disease recurring. Perhaps the patient himself may not be allowed to worry too much about that, but the clinicians looking after him are constantly aware of it.

\section{"A patient with an ileorectal anastomosis is living with the possibility of the disease recurring"- Mr Peter Bevan.}

I quite agree with $\mathrm{Mr}$ Hawley that with young patients it's always nice to preserve the rectum even if only for a few years and the younger they are the more abhorrent it is to remove the rectum as well. On the other hand, I now have a small group of patients who had an ileorectal anastomosis for some years though none of them actually reached the ten year period $\mathrm{Mr}$ Hawley mentioned. For one reason or another all of them had to have the rectum removed and an ileostomy performed. I think all of them have said to me that they wished they'd had the ileostomy earlier. They were far happier with a well controlled ileostomy than they were with an ileorectal anastomosis 
and a rather unreliable bowel habit. Another disadvantage of ileorectal anastomosis is the development of troublesome anal stenosis.

Mr HAwley: Probably there are no great differences between us: we've all had patients who've had an ileorectal procedure with a large number of bowel actions who have needed a permanent ileostomy and been much happier. But we also have patients who've had an ileostomy with a retained rectum and then a secondary ileorectal anastomosis done later. Ask those patients which they prefer: most of them have no hesitation in saying that their life is very much better with the ileorectal. It all depends on the patient and the functional result.

\section{Quality of life}

MR Bevan: I'm sure that's right. We have to consider the quality of life afterwards-so many of these patients are young and have got most of their life in front of them. I was impressed with $\mathrm{Mr}$ Jones's results from the point of bowel function: after the operation 13 of his 40 patients had three stools a day or fewer, and that's obviously compatible with a normal life. But a further 12 had four to six stools a day and a few even more. Surely a patient who has four or more stools a day is suffering under something of a social disadvantage ?

MR JONES: That is an exceptionally difficult question to answer because it is so very subjective. Furthermore, someone who has had a total colectomy with an ileorectal anastomosis in one stage has nothing to compare it with; and when the surgeon who has done the operation asks the questions he is likely to get a more favourable report from a patient perhaps than somebody who is independent. That was partly why in our series $\mathrm{Mr}$ Munro did all the interviewing: he was quite unknown to the patients. Even so, our impression was that even the people who were having four or six stools a day were still really quite reasonably satisfied. Certainly they were all holding down a job.

There obviously comes a time when the frequency of stool from an ileorectal procedure is such that the operation can no longer be seen as a good result. This is probably when a patient regularly has to get up at night to defaecate. As Mr Hawley wisely said, the assessment has to be set against the background of the whole lives the patients lead-whether they look normal, whether they feel normal, if they are thinking of getting married, if they are going to university, if they are starting an apprenticeship. When young people mix and make new friendships, the feeling of not having an ileostomy is very important.

MR BEVAN: On the other side of the fence patients who have a permanent ileostomy can benefit from modern ileostomy techniques and stoma therapy. I've got one patient who's had three children with an ileostomy; but I'm conscious of what you say, because we can't get away from the fact that an ileostomy is an abnormality.

MR HAWLEY: I think Mr Bevan's expectation of the number of bowel actions that these patients have is perhaps too few. At St Mark's the average (when Dr Newton looked at some of our cases a few years ago) was between four and six and a few patients with over that number were nevertheless content. It's if they get over ten bowel actions a day that they are not happy, particularly - as you've emphasised-if they have to get up at night.

\section{Whose decision?}

Chairman: Is there ever a difference of opinion between the surgeon and the patient about whether further surgery is needed? Objectively the result may be poor, with the patient having eight to ten bowel actions a day. On the other hand, he or she may not like the prospect of an ileostomy. Can or should one talk a patient into having an ileostomy in those circumstances?

Mr Bevan: This is a difficult problem. My own attitude is to indulge in as widespread consultation as possible-rather as in the old days we used to say we would never amputate a leg unless we had a second opinion.

MR HAWLEY: I think that's a wide approach. This is a big decision for the patient. It's not such a difficult decision if ther@ is the possibility of cancer intervening - and some patients kno that this is a possibility. So far as frequency of bowel action concerned, I think the patients make up their own minds. If a patient can cope happily with twelve bowel actions a day..whe am I to tell him that he can't ?

MR BEVAN: Duration of symptoms is another importare factor.

Mr HAWLEY: My experience is that those who have frequen bowel actions usually develop the problem early on. It's no: something which suddenly comes and goes-unless there is af attack of fairly acute colitis in the rectal stump. The sensitivity of the rectum is an index of the amount of active disease present and patients with frequent bowel actions usually are the one with active disease. This can be helped by treatment with salazoe pyrine and steroid enemas or suppositories.

MR JONES: Our experience has been that the bowel action in. some patients with a somewhat disappointing result for two ow

"When young people mix and make new friendships the feeling of not having an ileostomy is very important"-Mr Peter Jones.

three years has, with steady medical treatment, come down tæ three or four stools a day-and in the end an excellent resulpe It's important not to be too discouraged by the immediate state. On the other hand, another of our patients suddenly wen into a most acute episode of ulcerative proctitis-having had a excellent result for two years-and had to have almost af emergency excision of the rectum. So it can happen both ways

Coming back to Mr Bevan's point about when to excise the rectum, I agree that on the whole the patient probably decides Happily we've had very few of these. We've got one boy now who was operated on at the age of 16 when he was pretty il: He more or less refused to have an ileostomy and we decided on ileorectal anastomosis. He made a good recovery; but he always had four, five, or six stools a day. He grew, he married, he's had child, and now he's passing seven or eight stools a day and has just in the last six months or so had to get up at night. I saw him a fortnight ago in the outpatient department, and it was obvious that he was reaching the stage where the result was no longer adequate. But during that time he has married and conceive\& a child. If this lad had had a proctocolectomy we cannot be surfo that he would have been able to do that: men who have proctor colectomy rather rarely have trouble with impotence, but it is possibility.

\section{Sexual problems}

MR HAWLEY: This again to me is one reason why patient\$ shouldn't have their rectum taken out as an emergency, becaus in an urgent or emergency operation in the night, one tends t? excise the rectum in the same way as in an operation for cancer In Gut last year Professor John Lennard Jones published $\vec{\Phi}$ sexual survey of patients with ileostomies who had had rectat excisions and found that over $30 \%$ of them had sexual problems In the younger patients only one in 80 was completely impoten? but 17 further patients-that's 18 out of 80 had problems were only partially potent. This is a big problem; and I think can be overcome by removing the rectum electively and by doing an intersphincteric dissection and leaving all the fat in the pelvis and keeping close to the rectal wall.

Mr JONES: One other aspect is that the three of us talking 
here happen to see a lot of colitis. But the condition may turn up anywhere in an emergency, and the patient can't necessarily be transferred to a hospital where surgeons are doing excision of the rectum all the time. Your advice, Mr Hawley, to think hard in an emergency about possibly retaining the rectum is important-though undoubtedly sometimes proctocolectomy (as Peter Bevan has said) is the right operation.

MR BEVAN: When I referred earlier on to the high percentage of emergency proctocolectomies that have been part of my practice during the last 10 or 15 years I would agree that this is not an operation to be done in the middle of the night. When I said emergency I meant within a matter of days; if the patient doesn't settle with conservative treatment the operation should be done by the number one team. It needs the consultant and a first assistant of some experience and a full team working with full supportive facilities during the daytime.

\section{Variations on ileostomy}

MR BEVAN : Another important factor we have to bear in mind in deciding between an ileostomy and ileorectal anastomosis is, of course, the ileostomy itself. If ileostomy technique could be improved beyond the Brooke eversion ileostomy this again might be a factor that might persuade us not to attempt so many ileorectals. There have been two recent suggestions for improvement: the reservoir ileostomy patented by Nils Koch in Sweden, and the magnetic ileostomy. Unfortunately, neither seem to me entirely satisfactory.

MR HAWLEY: I think that the Koch ileostomy has been a considerable advance. It's not without its problems; but again patients who have experienced both have no hesitation in saying they prefer to have a reservoir with a continent valve. Now at St Mark's we're not great advocates of this operation; we've done 20 I think. The procedure is technically much more difficult, and the patient may develop many complications. We never press the idea on a patient; if he doesn't know about it we don't tell him. There have, however, been some young patients who have said "I'm not having my colon out unless this operation is done." At St Mark's we initially had poor results but they are getting better. The results of the last half dozen or so cases have really been successful.

MR Bevan: How many of your 20 cases are really truly continent?

MR HAWLEY: If the reservoir isn't totally continent the operation is a failure and they're better with a conventional ileostomy. They have to be totally continent. We have had two deaths in this series. One elderly patient, early in this series, perforated his pouch passing a tube seven months postoperatively. And another patient died with renal problems three months postoperatively. But, of the others being followed up, over half have a very good functional result. This means that they empty the pouch two or three times in 24 hours and are totally continent.

The whole problem with reservoir ileostomies is the valve. As has been mentioned, there is a way of overcoming the problem by putting a magnetic device around an ileostomy. I'm not very keen on it, the infection rate is high, and the devices are terribly heavy. One company is making a carbon fibre prosthesis which hardly weighs anything. If this works it may be the answer.

MR JONES: I haven't done an ileal reservoir for the simple reason that I have not been asked for it; and it has always seemed to me that it was sufficiently complicated for it not to be a thing you would wish on somebody who wasn't really very keen to have it. As Peter Bevan was saying earlier on, the present level of ileostomy care is really very high indeed-thanks partly to Professor Bryan Brooke and partly to the Ileostomy Association and all the pressure and hard work they've brought to improving apparatus and stoma care.

MR BEVAN: I would like to say one final thing. I don't think we started at opposite poles of the spectrum, but we have reached a large measure of agreement. I shall certainly continue my own tendency in the last few years-looking for a higher proportion of patients on whom to do an ileorectal anastomosis.

\title{
Process and Outcome
}

\section{Cost of carrying out clinical diagnostic tests}

\author{
D J BARNARD, J P BINGLE, C J GARRATT
}

British Medical fournal, 1978, 1, 1463-1465

\section{Summary and conclusions}

The total cost of performing diagnostic tests in a hospital laboratory during one year was assessed. The largest single item of expenditure was the cost of the salaries of the technical staff, while the cost of reagents (including

York District Hospital, York YO3 7HE

D J BARNARD, FIMLT, chief technologist, department of nuclear medicine J P BINGLE, DM, FRCP, consultant physician

Department of Chemistry, University of York, Heslington, York YO1 5DD

C J GARRATT, MA, PHD, senior lecturer radiopharmaceuticals) was relatively small. The total costs of carrying out diagnostic tests are much higher than is often recognised by those who request them. The use of relatively expensive, commercially available assay kits saves time and gives good value for money. It may be worth taking this into account when planning hospital budgets.

\section{Introduction}

We decided to investigate the importance of certain biochemical tests in clinical diagnosis and to assess the costs and value of these tests. We report our analysis of the costs to the National Health Service of biochemical tests performed in the department of Nuclear medicine, the District Hospital, York. Radiopharmaceuticals were used in all tests. The analysis was carried out 